Cahiers de la recherche sur les droits fondamentaux

$12 \mid 2014$

Droit et psychiatrie

\title{
La loi du 5 juillet 2011 : le point de vue d'un juge des libertés et de la détention
}

Thierry Hanouët

\section{(2) OpenEdition}

Édition électronique

URL : http://journals.openedition.org/crdf/1880

DOI : $10.4000 /$ crdf. 1880

ISSN : 2264-1246

Éditeur

Presses universitaires de Caen

Édition imprimée

Date de publication : 1 décembre 2014

Pagination : 47-52

ISBN : 978-2-84133-507-7

ISSN : $1634-8842$

Référence électronique

Thierry Hanouët, « La loi du 5 juillet 2011 : le point de vue d'un juge des libertés et de la détention », Cahiers de la recherche sur les droits fondamentaux [En ligne], 12 | 2014, mis en ligne le 01 octobre 2015, consulté le 12 février 2020. URL : http://journals.openedition.org/crdf/1880 ; DOI : 10.4000/crdf.1880 


\title{
La loi du 5 juillet 2011: le point de vue d'un juge des libertés et de la détention
}

\author{
Thierry HANOUËT \\ Président du tribunal de grande instance de Béthune
}

\author{
I. Une intervention judiciaire qui n'est pas nouvelle \\ II. L'accès au juge et son rôle avant le 1 1er août 2011 \\ III. Les conditions du contrôle judiciaire depuis la loi du 5 juillet 2011, \\ modifiées par la loi du 27 septembre 2013 \\ A. Le lieu de l'audience \\ B. La publicité des débats \\ C. Le délai de saisine \\ D. Le recours à l'avocat et le déroulement de l'audience \\ E. L'office du juge \\ IV. Le contrôle de la régularité des décisions administratives
}

Invité à participer au colloque du 15 novembre 2013 en tant que juge des libertés et de la détention (JLD), il convient de préciser au préalable que j'avais largement participé à la préparation de la mise en œuvre de la loi no $2011-803$ du 5 juillet 2011 relative aux droits et à la protection des personnes faisant l'objet de soins psychiatriques et aux modalités de leur prise en charge. Je n'exerce plus les fonctions de juge des libertés, mais suis plutôt en charge de veiller au bon fonctionnement du service qui gère ce contentieux au sein de la juridiction que je préside dans le Pas-de-Calais. À ce titre, l'évolution du dispositif légal qui résulte de la loi $n^{\circ} 2013-869$ du 27 septembre 2013 modifiant certaines dispositions issues de la loi du 5 juillet 2011 est loin de me laisser indifférent. Si l'on se concentre sur les éléments les plus essentiels, il est possible de mettre en exergue quatre points: une intervention judiciaire qui n'est pas nouvelle (I); l'accès au juge et son rôle avant le $1^{\text {er }}$ août 2011 (II); les conditions du contrôle judiciaire depuis la loi du 5 juillet 2011, modifiées par la loi du 27 septembre 2013 (III) ; le contrôle de la régularité des décisions administratives (IV).

\section{Une intervention judiciaire qui n'est pas nouvelle}

Jusqu'à une époque récente, le président du tribunal de grande instance était compétent pour statuer sur le sort des malades psychiatriques. Le JDL, créé en $2000^{1}$, lui a succédé.

1. Loi $\mathrm{n}^{\circ} 2000-516$ du 15 juin 2000 renforçant la protection de la présomption d'innocence et les droits des victimes. 
On comprend de prime abord qu'il s'agit d'un juge qui a une activité pénale, puisqu'il décide de la détention des personnes qui ont été mises en examen par le juge d'instruction et statue sur le renouvellement des mesures de détention provisoire et sur les demandes de mise en liberté. Mais ce JLD a aussi une activité civile: on songe à l'important contentieux de la rétention des étrangers et, marginalement jusqu'au $1^{\text {er }}$ août 2011, il recevait la visite de quelques patients des établissements psychiatriques qui contestaient la prise en charge offerte par les services de soins.

Un seul et même juge est en charge de décider de l'incarcération, de la poursuite de la rétention ou de la poursuite de l'hospitalisation. On comprend la volonté de cohérence qui préside à ce choix et, en même temps, on ne peut s'empêcher de penser que le caractère purement civil de la question de la poursuite des soins contraints puisse être plus ou moins brouillé par l'intitulé fonctionnel, juge des libertés et de la détention, surtout auprès d'un public fragile. De plus, le JDL est un magistrat qui, au quotidien, décide de l'incarcération pour des faits souvent graves et on peut imaginer qu'il lui est moins difficile qu'à d'autres, compte tenu de sa pratique, de maintenir l'hospitalisation d'un patient contre sa volonté, alors que la mesure est en principe motivée par la nécessité du soin et qu'elle est prise dans l'intérêt supérieur du malade.

Il ne faut pas non plus mésestimer l'importance du rôle du procureur de la République qui doit être informé des mesures d'hospitalisations forcées. Sans entrer dans le détail, on peut souligner l'éminence de la mission du procureur de la République, rappelée par des circulaires anciennes du 8 décembre 1938 et du 21 juillet 1952, dont il résultait qu'une enquête pouvait être ordonnée hors les cas où la maladie était évidente. Cette enquête pouvait avoir pour objectif de déterminer:

- les circonstances qui avaient motivé l'internement. Il s'agissait principalement d'entendre en toute discrétion les membres de la famille;

- l'état de fortune de l'intéressé, au cas où on serait confronté à une tentative de spoliation par les proches;

- l'existence d'un procès dans lequel était engagé l'aliéné.

Le procureur avait également la faculté de visiter ou de faire visiter le malade sans que l'entourage en soit informé. Il était également censé visiter une fois par trimestre les établissements publics ou privés accueillant des malades soignés pour troubles mentaux. Une circulaire de 1906 estimait que «ces visites ne sauraient évidemment être de simples formalités, car ainsi comprises, elles seraient indignes du caractère des magistrats qui les accompliraient ». Elles devaient s'étendre à l'ensemble des locaux hospitaliers, permettre de vérifier les registres et de rencontrer les malades et plus particulièrement ceux qui avaient adressé des réclamations à l'autorité judiciaire. Il était même donné un modèle de lettre: «J'ai rencontré Monsieur X qui m'a paru avoir recouvré ses facultés mentales et en faveur duquel je me propose de saisir le Président du tribunal de grande instance».

La loi nouvelle a maintenu au procureur de la République toute sa place. Il peut former un recours contre la décision de soins psychiatriques quelle qu'en soit la forme, et il donne son avis à l'audience ou prend des conclusions écrites. Les articles L. 3212-5 et L. 3213-9 du Code de la santé publique (CSP) prévoient que le procureur de la République est informé de toute décision d'admission d'une personne en soins psychiatriques. En vertu de l'article L. 3211-12 $7^{\circ} \mathrm{CSP}$, le procureur de la République peut à tout moment saisir le JDL aux fins qu'il ordonne la mainlevée immédiate d'une mesure de soins psychiatriques. Il prend des conclusions écrites ou est présent à l'audience.

On voit donc que le procureur de la République est lui aussi garant des libertés publiques, ce qui ne devrait étonner personne, même si cette institution est plutôt identifiée comme une autorité de poursuite en matière pénale.

\section{L'accès au juge et son rôle avant le $1^{\text {er }}$ août 2011}

Il convient pour l'essentiel de se reporter à l'article L. 321112 CSP (ancien):

Une personne hospitalisée sans son consentement ou retenue dans quelque établissement que ce soit, public ou privé, qui accueille des malades soignés pour troubles mentaux, son tuteur si elle est mineure, son tuteur ou curateur si, majeure, elle a été mise sous tutelle ou en curatelle, son conjoint, son concubin, un parent ou une personne susceptible d'agir dans l'intérêt du malade et éventuellement le curateur à la personne peuvent, à quelque époque que ce soit, se pourvoir par simple requête devant le juge des libertés et de la détention du tribunal de grande instance du lieu de la situation de l'établissement qui, statuant en la forme des référés après débat contradictoire et après les vérifications nécessaires, ordonne, s'il y a lieu, la sortie immédiate.

Une personne qui a demandé l'hospitalisation ou le procureur de la République, d'office, peut se pourvoir aux mêmes fins.

L'expérience montre que dans la très grande majorité des cas, qu'il s'agisse d'hospitalisations à la demande d'un tiers ou d'hospitalisations d'office, le juge était saisi par la personne hospitalisée elle-même. On peut y voir l'indication qu'a priori elle avait reçu l'information nécessaire sur le lieu de soins, même si des questions demeurent sur ce point. On sait que le degré d'information et le moment de celle-ci sont susceptibles de varier notablement d'un secteur psychiatrique à l'autre. Certains considèrent qu'il faudrait attendre que la personne soit en état de la recevoir, ce qui pourrait reporter d'autant l'exercice effectif de ses droits. La demande de mainlevée par un proche est quantitativement marginale alors que les textes permettent la saisine par toute personne ou presque. 
Le parquet est avisé, la personne hospitalisée est convoquée et reçue au cabinet du JLD, avec le personnel soignant qui l'accompagne. L'audience consiste en un échange oral de trente à quarante-cinq minutes. Le juge interroge la personne en commençant par lui rappeler les termes de sa requête, l'interroge sur son histoire médicale, sa vie professionnelle, familiale, ses doléances. Dans le respect du principe du contradictoire elle est confrontée aux certificats médicaux figurant au dossier et transmis par l'établissement de soins. En dehors des situations de déni, pur et simple («je ne suis pas fou ce sont les médicaments qui me rendent malades»), on peut faire le constat que de nombreux requérants critiquent les soins dont ils font l'objet, au moins sous l'angle des effets jugés indésirables (somnolence, ralentissement psychique, perte de libido, impossibilité de travailler). Ils sont en demande d'arrêt du traitement ou, pour le moins, d'aménagement des soins. On peut percevoir une forme de lassitude, ils peuvent recevoir des soins depuis plusieurs années et ils en ont assez! Souvent ils souhaitent simplement rentrer chez eux, d'autres expriment l'ennui: «on n'a rien à faire!». Il est relativement fréquent que, sur un entretien de trente à quarante-cinq minutes, le discours soit émaillé de propos ou de projets qui alertent sur l'inscription dans la réalité de la personne hospitalisée (propos délirants, délire mystique ou apocalyptique).

Le greffier établit une note d'audience relatant les propos échangés, note qui est versée au dossier. L'affaire est normalement mise en délibéré à quelques jours pour être rédigée, formalisée, puis notifiée par fax au patient, à l'établissement et/ou au préfet.

Le juge peut toutefois s'interroger sur la pertinence de la mesure d'hospitalisation forcée quand l'entretien ne lui a pas permis de déceler chez son interlocuteur de signes de pathologie évidents, au sens où les pièces médicales semblent ne pas correspondre à la personne qu'il a en face de lui. Dans ce cas, sauf décision de mainlevée immédiate, il est recouru à l'expertise psychiatrique qui est confiée à un médecin expert psychiatre exerçant hors l'établissement ou tout simplement retraité. Une nouvelle audience est programmée à réception du rapport de l'expert pour en notifier les conclusions à la personne hospitalisée et en reprendre avec elle les termes.

Aucune expertise n'a jamais conclu que l'individu ne présentait pas de graves troubles, nous n'avons donc jamais eu le sentiment d'être confronté au péril de la séquestration arbitraire. En revanche, il a été assez régulièrement indiqué que l'hospitalisation forcée pourrait être levée à court / moyen terme, après le succès de quelques sorties d'essai. Au fond, la critique de l'hospitalisation forcée, devant le juge judiciaire, réside dans le fait qu'elle se prolonge pour garantir la poursuite des soins, alors que la personne hospitalisée est un peu plus pressée que son médecin. En ce qui me concerne, la logique de protection des personnes m'a plutôt conduit à soutenir le point de vue médical auprès de la personne requérante pour l'inciter à une alliance thérapeutique.

On doit, pour être complet, souligner que parfois le mieux est l'ennemi du bien et que les délais pour statuer imposés par le décret du 22 mai 2010 n’ont pas amélioré le traitement de ce contentieux. Il a fallu abandonner la pratique qui consistait à ordonner une expertise dès la saisine, ce qui pourtant semblait une garantie pour le requérant et une information précieuse pour le juge. Un autre argument pour abandonner cette pratique, peu avouable lui, tenait au coût de ces expertises eu égard aux contraintes budgétaires pesant sur les frais de justice. Mais surtout, le manque de disponibilité des experts psychiatres a empêché de statuer dans le délai de vingt-cinq jours imposé par le décret (douze jours si une expertise n'est pas ordonnée).

Statistiquement, on peut considérer que $10 \%$ des requêtes environ ont abouti à une mainlevée judiciaire de la mesure d'hospitalisation forcée. Par ailleurs, on peut observer que, de manière habituelle, des requêtes ne donnaient lieu à aucun débat, la personne étant sortie de l'établissement de soins avant même sa comparution devant le juge.

\section{Les conditions du contrôle judiciaire depuis la loi du 5 juillet 2011, modifiées par la loi du 27 septembre 2013}

Le Conseil constitutionnel a déclaré le 26 novembre 2010 non conforme à la Constitution le maintien d'une hospitalisation forcée au-delà de quinze jours, sans l'intervention d'un juge judiciaire, garant de la liberté individuelle ${ }^{2}$. Actuellement, la mise en œuvre de la loi de 2011 s'articule autour de trois périodes de temps:

- les soixante-douze heures de l'évaluation initiale;

- le délai de quinze jours, avec contrôle de plein droit, délai qui sera de douze jours à compter du $1^{\mathrm{er}}$ septembre 2014;

- le délai de six mois, avec contrôle de plein droit.

Désormais, le JLD peut être saisi selon deux modalités principales: par l'exercice d'un recours facultatif contre la mesure de soins aux termes des dispositions de l'article L. 3211-12 CSP et au titre du contrôle de plein droit de l'article L. 3211-12-1 du même Code. L'examen du recours facultatif se fait dans les conditions déjà décrites, le dispositif antérieur à la réforme est globalement reconduit. Le contrôle de plein droit présente quelques particularités, tenant au lieu de l'audience (A), à la publicité des débats $(B)$, au délai de saisine $(C)$, au recours à l'avocat et au déroulement de l'audience (D) et à l'office du juge (E). 


\section{A. Le lieu de l'audience}

Si habituellement, et sauf exceptions, le travail juridictionnel se fait au tribunal, la loi de 2011 offrait trois possibilités: tenir l'audience au cabinet du JLD, se déplacer et se rendre sur les lieux d'hospitalisation ou recourir à la visioconférence. Cette dernière possibilité pouvait poser quelques difficultés: elle pouvait être refusée par le patient, au moins parce qu'il ne veut pas être filmé. Par ailleurs, les soignants pouvaient estimer que son utilisation était contre-indiquée pour des raisons médicales (et ce même si dans de nombreuses juridictions les audiences se tiennent en visioconférence de manière systématique, sans que soient évoquées de difficultés). Enfin, elle entraînait une perte très importante de la qualité de l'échange entre le juge et le patient.

Ce dispositif a été totalement remanié par la loi du 27 septembre 2013 (applicable sur ce point à compter du $1^{\mathrm{er}}$ septembre 2014) qui dispose que désormais l'audience se tiendra en priorité dans une salle d'audience spécialement aménagée sur l'emprise de l'établissement d'accueil qui sera attribuée au ministère de la Justice et dédiée aux audiences du JLD, le recours à la visioconférence étant lui purement et simplement supprimé. La salle devra être agencée de façon à assurer la clarté et la sincérité des débats, la sécurité, l'accès au public. On devra donc comprendre que l'on est bien dans une salle d'audience judiciaire. En l'absence de salle conforme dans l'établissement, l'audience devra se tenir au tribunal de grande instance. En revanche, en appel, le débat se tient obligatoirement au siège de la juridiction.

\section{B. La publicité des débats}

C'est là une nouveauté très importante de la loi de 2011, dont on peut dire qu'elle paraît choquante à beaucoup et en particulier à nombre de soignants qui y voient une atteinte au respect de la vie privée des malades. La publicité doit s'entendre comme une garantie pour le justiciable. C'était sans doute devenu une nécessité que de la prévoir pour se mettre en conformité avec les impératifs posés par l'article 6 de la Convention européenne des droits de l'homme, qui pose le principe que toute personne a droit à ce que sa cause soit entendue publiquement et que le jugement soit rendu en audience publique, sauf cas particuliers (intérêt de la moralité, de l'ordre public, sécurité nationale, intérêts des mineurs, protection de la vie privée).

Il est vrai que ce qui va se dire à l'audience a manifestement trait à la sphère personnelle, à l'intime. Pour autant, la possibilité du débat public demeure une protection pour les usagers dans la mesure où elle impose aux professionnels d'être vigilants sur la forme et sur le fond. Rien que pour cela elle est très utile.

La possibilité de limiter la publicité est élargie par la loi du 27 septembre 2013 qui prévoit que le juge pourra restreindre la publicité sur demande d'une des parties.

Les débats devront se tenir de plein droit en chambre du conseil si la demande émane de la personne qui fait l'objet de soins psychiatriques.

\section{Le délai de saisine}

S’agissant du délai de quinze jours, le juge peut être saisi jusqu'au douzième jour, il lui reste donc trois jours pour organiser l'audience, fixer le lieu, convoquer, réunir les pièces et préparer les documents qui seront notifiés à l'issue des débats. Ce délai est ramené à douze jours par la loi nouvelle, sa saisine devant intervenir dans un délai de huit jours. Cette mesure prend effet au $1^{\mathrm{er}}$ septembre 2014.

S'agissant du délai de six mois, le juge doit être saisi huit jours avant l'expiration du délai. La loi nouvelle prévoit que la saisine doit intervenir quinze jours avant l'expiration du délai. Cette mesure prend effet au 15 mars 2014. La demande tardive est irrecevable.

\section{Le recours à l'avocat et le déroulement de l'audience}

L'assistance de l'avocat est désormais obligatoire. Il assiste la personne hospitalisée. La personne qui ne peut être entendue pour des raisons médicales est représentée par un avocat.

L'audience se déroule comme déjà indiqué, le débat changeant toutefois de nature puisqu'il ne s'agit plus d'examiner une demande d'une personne hospitalisée, mais celle de l'établissement ou du préfet. Mais le juge ne voit pratiquement plus que des patients fraîchement hospitalisés, dans un temps proche de la crise qui a conduit à la mesure. Il me semble toujours souhaitable de préciser au patient le cadre juridique de notre intervention, de reprendre avec lui ce pourquoi il est dans l'établissement (s'il s'en souvient), son parcours de soins, s'assurer de ce qu'il reconnaît ou non souffrir d'une pathologie, et le confronter au certificat du médecin qui vient nécessairement soutenir la requête. Cet entretien sera plus ou moins approfondi, en fonction de ce que l'on peut percevoir de l'état de santé du patient, de son anxiété, de son agressivité, de sa capacité à échanger, de son niveau de sédation. Il faudra être, en tout état de cause, respectueux et rassurant. Au fond il convient de s'adresser au patient hospitalisé comme on aimerait qu'on s'adresse à nous.

\section{E. L'office du juge}

Il s'agit donc de vérifier que l'atteinte à la liberté du patient résultant de la mesure d'hospitalisation forcée est adaptée, nécessaire et proportionnée aux objectifs poursuivis:

- l'état mental impose des soins immédiats assortis d'une surveillance médicale constante justifiant une hospitalisation complète, aux termes de l'article L. 3212-1 CSP;

- l'état mental impose des soins et les troubles du patient sont de nature à compromettre la sûreté des personnes ou à porter atteinte, de façon grave, à l'ordre public, aux termes de l'article L. 3213-1 CSP. 


\section{Le contrôle de la régularité des décisions administratives}

Depuis le $1^{\text {er }}$ janvier 2013, le contrôle des décisions administratives a été largement remanié. En vertu de l'article L. 3216-1 CSP :

La régularité des décisions administratives prises en application des chapitres II à IV du présent titre ne peut être contestée que devant le juge judiciaire.

Le juge des libertés et de la détention connaît des contestations mentionnées au premier alinéa du présent article dans le cadre des instances introduites en application des articles L. 3211-12 et L. 3211-12-1. Dans ce cas, l'irrégularité affectant une décision administrative mentionnée au premier alinéa du présent article n'entraîne la mainlevée de la mesure que s'il en est résulté une atteinte aux droits de la personne qui en faisait l'objet.

Lorsque le tribunal de grande instance statue sur les demandes en réparation des conséquences dommageables résultant pour l'intéressé des décisions administratives mentionnées au premier alinéa, il peut, à cette fin, connaître des irrégularités dont ces dernières seraient entachées.

De la lecture des débats parlementaires, il ressort que le législateur a entendu prendre en compte la difficulté pour l'usager souffrant de troubles mentaux que représentait la dualité des ordres de juridictions. En effet, la situation de l'usager était difficile puisque s'il entendait contester la régularité de la procédure d'admission en soins, il devait s'adresser au juge administratif, mais s'il entendait remettre en cause le bien-fondé de la mesure, il devait saisir le juge judiciaire. Sont désormais visées toutes les situations de soins psychiatriques sans consentement, quelle que soit la date de la décision contestée, la juridiction administrative demeurant compétente pour les recours dont elle était saisie avant le $1^{\mathrm{er}}$ janvier 2013. Mais le transfert de compétence est assez limité en réalité car il ne s'agit que de contrôler la régularité des décisions qui ont abouti aux mesures de soins contraints prévues par les chapitres II à IV du titre du CSP intitulé «Modalités de soins psychiatriques», soit les décisions prises en cas d'admission à la demande d'un tiers ou en cas de péril imminent (chapitre II), en cas de décision du préfet (chapitre III), ou pour les personnes détenues (chapitre IV). Tous les autres contentieux demeurent de la compétence des juridictions administratives. On peut citer par exemple la question des transferts d'un établissement à un autre (ce point est discuté), la contestation du traitement médical ou encore les refus de prononcer l'hospitalisation ou le refus de transfèrement.

Deux conditions doivent être réunies pour aboutir à la mainlevée de la mesure:

1. La décision administrative doit être irrégulière, au sens où elle n'a pas été prise conformément aux procédures prévues par la loi et les règlements. On perçoit donc que les hypothèses d'irrégularité de la décision administrative peuvent être nombreuses:
- l'incompétence de l'auteur: les articles L. 3213-1 et L. 3212-1 CSP prévoient que sont compétents pour prononcer l'admission en soins psychiatriques le représentant de l'État dans le département et le directeur de l'établissement spécialisé. Ils peuvent déléguer leur compétence, mais la délégation écrite doit au moins mentionner le nom et la fonction de l'agent délégataire, la nature des actes délégués, voire leur durée. Elle doit être publiée (on pense à un affichage dans l'établissement) (L. 6143-7, D. 6143-33 à 35). A priori, il n'y a donc pas de régularisation possible;

- les vices de procédure: ceux-ci sont multiples, mais pour simplifier on peut considérer que l'absence de notification des droits constitue un vice de procédure (possibilité de saisir le JLD ou de saisir la commission de contrôle des soins psychiatriques ou possibilité de communiquer avec les autorités telles que le préfet, le président de la République, le président du tribunal de grande instance...). Il en est de même de l'absence de notification de la décision imposant les soins, de l'absence des certificats prévus par la loi, de la non-information du procureur ou de telle autorité, du défaut de qualité du tiers (si la personne n'est pas un membre de la famille ou une personne justifiant de l'existence de relations avec le malade avant la demande de soins et lui donnant qualité à agir dans l'intérêt de celui-ci, si la personne est un soignant de l'hôpital spécialisé, ce qui ne serait pas le cas de l'assistante sociale du secteur psychiatrique, même salariée par l'hôpital, si la personne est malveillante, par exemple une épouse, alors que le couple est en instance de divorce et en relations très conflictuelles). Il convient d'y ajouter le respect du principe du contradictoire qui s'impose hors procédure d'urgence. L'intéressé doit avoir été mis à même de présenter ses observations ou il doit avoir été constaté l'impossibilité de les recueillir. Ce principe ne s'applique pas en cas de troubles graves à l'ordre public, la décision d'hospitalisation s'imposant au vu des circonstances (danger pour autrui et nécessité de soins, par exemple une personne délirante qui brandit une machette sur la voie publique);

- les vices de forme: l'auteur de la décision doit être identifiable, la décision doit mentionner les nom et prénom et la qualité de celui-ci. L'acte doit être lisible, ce qui présente un intérêt pratique et impose de ne pas se satisfaire de copies de documents illisibles. Mais le principal vice de forme est le défaut de motivation. Si le principe demeure en droit administratif que la motivation est facultative, il est constant que par l'effet de la jurisprudence et de la loi, s'est imposée la nécessité de motiver les décisions administratives dans de nombreux domaines. C'était déjà le cas en matière d'hospitalisation d'office des personnes atteintes de troubles mentaux et cela reste une exigence de la loi nouvelle pour l'autorité compétente de 
motiver sa décision en fait et / ou en droit au regard des conditions posées par la loi pour chacun des cas de recours. Ainsi, lorsque les soins sont imposés par le préfet on doit retrouver dans la motivation l'indication de l'existence de troubles mentaux nécessitant des soins et les éléments laissant craindre un danger pour la personne ou les tiers ou un risque d'atteinte grave à l'ordre public. Le Conseil d'État, dans son arrêt Deslandes du 9 novembre 2001, a déjà proposé une réponse en indiquant que:

L'autorité administrative, lorsqu'elle prononce ou maintient l'hospitalisation d'office d'un aliéné, doit indiquer dans sa décision les éléments de droit ou de fait qui justifient cette mesure, que si elle peut satisfaire à cette exigence de motivation en se référant au certificat médical circonstancié qui doit être nécessairement établi avant la décision préfectorale, c'est à la condition de s'en approprier le contenu et de joindre ce certificat à la décision ${ }^{3}$.

Il va de soi que le juge judiciaire aura d'autant plus de difficulté à maintenir la mesure de soins contraints, s'il ne trouve pas dans les pièces du dossier les éléments lui permettant de motiver sa décision. Or, le sentiment général est que c'est ce qui fait véritablement difficulté.

2. L'irrégularité en cause constitue une atteinte aux droits de la personne qui faisait l'objet de soins contraints. L'intention du législateur était qu'il devait être évité qu'une irrégularité purement formelle et dénuée de tout impact sur le bien-fondé de la décision, puisse entraîner la mainlevée de la mesure. Pas de nullité sans grief donc. La jurisprudence définira par touches successives ce que sera ce critère.

On peut déjà observer que les restrictions apportées à l'exercice des libertés individuelles de la personne qui fait l'objet de soins psychiatriques doivent être adaptées, nécessaires et proportionnées à son état mental et à la mise en œuvre du traitement requis. Sa dignité doit être respectée et sa réinsertion recherchée.

L'irrégularité n'est pas qualifiée, elle n'est pas nécessairement grave. Le juge peut la constater sans pour autant considérer qu'elle porte atteinte aux droits.

L'irrégularité n'a pas obligatoirement affecté le bienfondé de la mesure, sinon il n'y a plus de contrôle de régularité (parce qu'alors le contrôle du juge se confond avec la vérification du bien-fondé de la mesure).

L'irrégularité peut avoir été commise à tout moment, mais dès que le juge la constate il peut prononcer la mainlevée de la mesure de soins.
Si l'on se place dans une logique de défense large des droits fondamentaux, on doit alors considérer que même si la mesure d'hospitalisation est fondée sur le fond, l'irrégularité de la procédure affecte les droits de la personne, sa liberté tout particulièrement, et lui cause donc un préjudice. À terme, pourrait s'imposer la distinction entre irrégularités qui font grief et qui donc emportent nécessairement mainlevée de la mesure de soins, abstraction faite de la pathologie du malade, et irrégularités formelles sans effet sur la mesure de soins (distinction classique en procédure civile et désormais à l'œuvre aussi en droit administratif depuis peu pour éviter la paralysie de l'action publique pour des problèmes de virgules).

Les conséquences: le JLD prononce la mainlevée de la mesure ou rejette la demande. Dans les deux cas il rend une décision motivée. Il peut laisser un délai de vingt-quatre heures à l'administration pour organiser un programme de soins. Un recours suspensif est possible. Mais le JLD ne prononce pas l'annulation de la décision administrative. Dès lors, si un usager veut obtenir l'annulation de la décision critiquée, il devra saisir le juge administratif.

En conclusion, on peut relever que l'hospitalisation en hôpital psychiatrique n'est pas partout la règle. Sans aller bien loin, on peut indiquer que l'Angleterre a fermé ses hôpitaux psychiatriques il y a plus de vingt ans pour investir fortement dans les services ambulatoires (il y a dix fois moins de lits qu'en France avec un budget équivalent au budget français). Elle a créé des prisons-hôpitaux sous contrôle judiciaire, les autres lits sont à l'hôpital général avec des unités sécurisées. Il y a moitié moins de psychiatres qu'en France (6 ooo pour 13000 en France) et personne ne dit qu'il en manquerait! Le même constat peut être effectué concernant l'Italie, où les hôpitaux psychiatriques ont fermé effectivement en 1995 (réforme de 1980) avec un redéploiement vers l'ambulatoire (12 00o lits au lieu de 58000 comme en France et 1600 lits judiciaires qui ne sont pas tous occupés!; on compte par ailleurs 7000 psychiatres).

J'observe enfin une évolution considérable entre le législateur de 1990 qui s'opposait à la judiciarisation de ce contentieux au motif qu'elle stigmatiserait le patient en l'assimilant à un délinquant, alors qu'en 2011 c'est la présence de ce même juge qui est apparue comme une garantie, sous l'incitation du Conseil constitutionnel, il faut le souligner. On ne fait que découvrir en France, ce qui se pratique assez largement ailleurs en Europe avec beaucoup de différences procédurales selon les pays. 